\title{
REGRESSION OF MAJOR RECURRENT APHTHOUS ULCERATIONS USING A COMBINATION OF INTRALESIONAL CORTICOSTEROIDS AND LEVAMISOLE: A CASE REPORT
}

doi: $10.1590 /$ S1807-59322010000600015

Bruna Lavinas Sayed Picciani, Geraldo Oliveira Silva-Junior, Davi Silva Barbirato, Ruth Tramontani Ramos, Marilia Heffer Cantisano

\section{INTRODUCTION}

Recurrent aphthous ulcerations (RAU) are common oral lesions that affect approximately $10 \%$ to $20 \%$ of the population. The disease is characterized by recurrent and painful ulcerations of the oral non-keratinized mucosa, which measure from 3 to $10 \mathrm{~mm}$ in diameter and heal without scarring in 7 to 14 days. ${ }^{1}$ The etiology of RAU remains unknown, but there is increasing evidence that its development has an immunogenic basis. The search for its cause should concentrate on disorders associated with immunoderegulation, decreased mucosal barriers, or conditions that elevate the antigenic stimulus. Major RAU have irregular margins, frequently exceed $10 \mathrm{~mm}$ in size, and typically leave scars. ${ }^{2}$

There have been many attempts over the years to find an effective treatment for RAU. Because the etiology of RAU remains unknown, its treatment consists of therapeutic measures to suppress its symptoms rather than bringing about a definitive cure. The therapeutic choice depends on the severity of the disease, which is measured by the frequency of ulcer recurrence, the number of ulcers, their location and duration, and the level of associated orofacial pain. ${ }^{3,4}$ Cases of major RAU that are characterized by pain, fever, and dysphagia and that are recurrent usually require systemic therapy. Several systemic drugs have been used to treat major RAU, including systemic corticosteroids, dapsone, colchicine, thalidomide, pentoxifylline, low-dose interferon- , and levamisole. ${ }^{2,5}$

Department of Diagnosis and Surgery, Stomatology, School of Dentistry, State University of Rio de Janeiro - Rio de Janeiro/RJ, Brazil.

Email: mcantisano@terra.com.br

Tel.: 5521 2587-6457
The use of systemic corticosteroids and immunomodulating agents has been the mainstay of treatment for major RAU. Intralesional steroid injections, which contain betamethasone dipropionate and betamethasone disodium phosphate, combined with levamisole therapy may successfully treat the large ulcers associated with major RAU. ${ }^{6}$

This paper presents a case report of major RAU where we tested the therapeutic efficacy of a treatment combining an intralesional corticosteroid and a drug used to modulate immune responses.

\section{CASE REPORT}

A 30-year-old woman presented a 15-year history of successive episodes of multiple major recurrent ulcers in the oral mucosa and tongue region of the mouth, which left scars after lesion regression. During the patient's anamnesis, she reported having had these episodes since adolescence and having already been treated with corticosteroids, but without success. Clinical examination revealed symptomatic ulcers with perilesional erythematous halos that were covered with a pseudo membrane and located in the jugal mucosa and on the right side border of the tongue, exceeding 10 $\mathrm{mm}$ in diameter (Fig. 1a). There were also areas of scarring on the right jugal mucosa. A clinical diagnosis of major RAU was made on the basis of the history and the clinical examinations. The other aspects of the patient's medical history and family medical history were insignificant. Laboratory tests including a blood count analysis were performed to determine the levels of folic acid, iron, ferritin, as well as vitamins B2, B6, and B12, all of which showed normal results.

The patient was subjected to a therapeutic consisting 

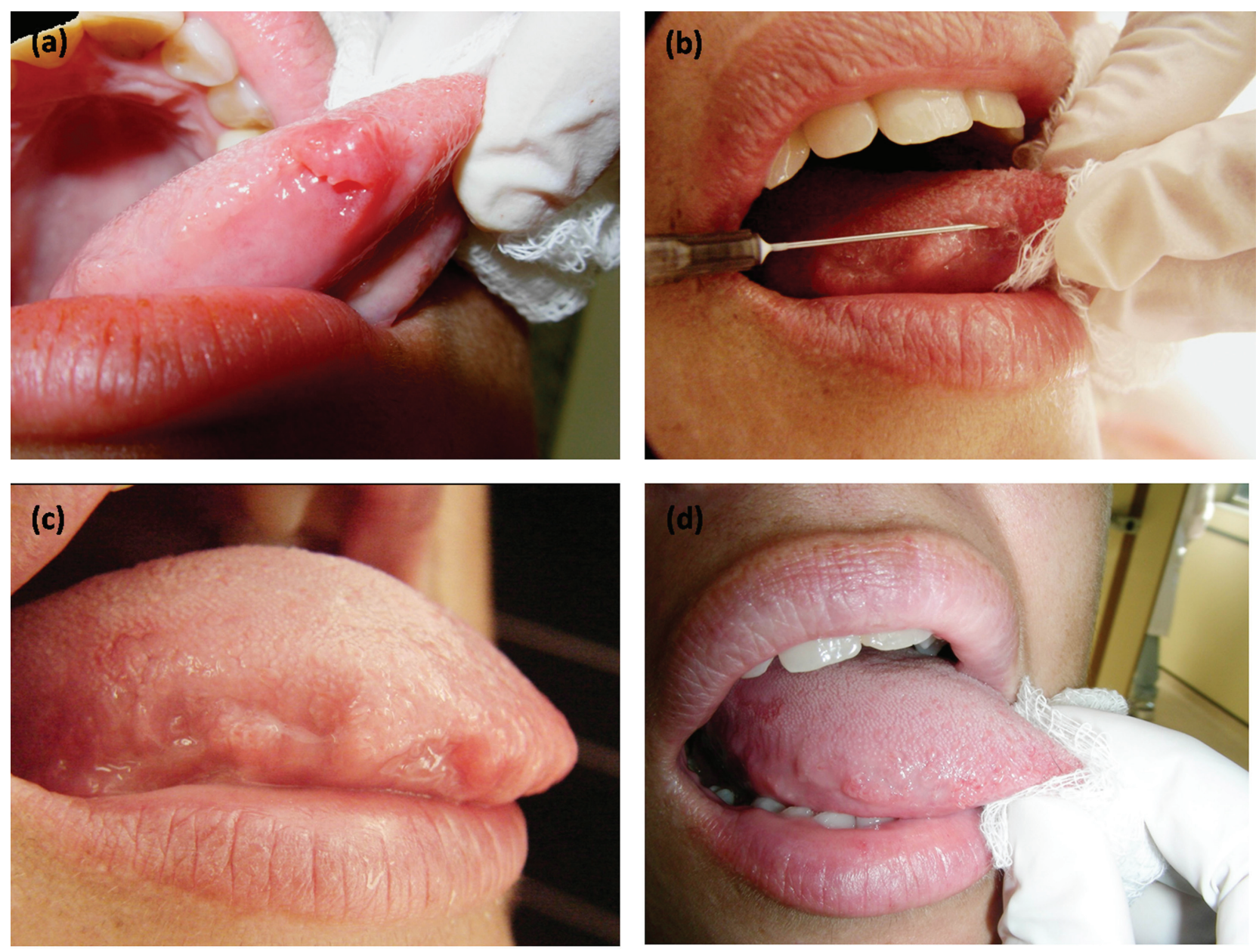

Figure 1 - (a) Major recurrent aphthous ulceration on the right border of the tongue; (b) intralesional injection of corticosteroids; (c) partial regression of the lesions was achieved one week after the administration of intralesional corticosteroids; (d) the major recurrent aphthous ulceration was resolved and no recurrence was observed after four weeks treatment with levamisole.

of prednisone (15 $\mathrm{mg}$ per day) and a topical mouthwash containing dexamethasone (20 mg per day) for two weeks, which was unsuccessful. Thus, a regimen was proposed consisting of an intralesional injection of betamethasone dipropionate $(5 \mathrm{mg} / \mathrm{ml})$ and betamethasone disodium phosphate $(2 \mathrm{mg} / \mathrm{ml})$ (Fig. 1b). After the first application, there was a partial regression of the lesions. Only one application was needed to bring about partial healing of the ulcers (Fig. 1c). To reduce the frequency of ulceration, levamisole hydrochloride $(150 \mathrm{mg})$ was administered once a week for one month. Despite considerable improvement in the patient's status, it has not yet been possible to precisely establish the etiologic agent. There was no recurrence during the 6-month follow-up period (Fig. 1d).

\section{DISCUSSION}

The clinical features, etiology, and treatment of major recurrent aphthous ulcerations have been discussed. The primary goals of administering therapy for major RAU are to bring about relief from pain, reduction in ulcer duration, and the restoration of normal oral function. Secondary goals include a reduction in the frequency and severity of recurrences and the maintenance of remission. Topical medications, such as corticosteroids, can achieve the primary goals but have not been shown to alter recurrence or remission rates. Systemic medications can be combined with the topical therapy to achieve the secondary goals. ${ }^{7,8}$ Levamisole, an antihelminthic drug that can modulate immune responses, has been used successfully as a monotherapy and as an adjunct to treatment in a variety of diseases. Because of its modulating effects on immune responses, levamisole has been used in a wide range of diseases with varying degrees of success. It has also been used in combination with corticosteroids to treat a number of mouth dermatologic disorders, such as major RAU. ${ }^{9,10}$

The therapeutic method used in this case provided significant relief from symptoms and decreased the rate of recurrence. Additional studies are needed to evaluate the full potential of levamisole in oral dermatological diseases. 


\section{REFERENCES}

1. Natah SS, Konttinen YT, Enattah NS, Ashammakhi N, Sharkey KA, Hayrinen-Immonen R. Recurrent aphthous ulcers today: a review of the growing knowledge. Int J Oral Maxillofac Surg. 2004;33:221-34.

2. Burruano F, Tortorici S. [Major aphthous stomatitis (Sutton's disease): etiopathogenesis, histological and clinical aspects]. Minerva Stomatol. 2000;49:41-50.

3. Munoz-Corcuera M, Esparza-Gomez G, Gonzalez-Moles MA, Bascones-Martinez A. Oral ulcers: clinical aspects. A tool for dermatologists. Part I. Acute ulcers. Clin Exp Dermatol. 2009;34:289-94.

4. Ship JA. Recurrent aphthous stomatitis. An update. Oral Surg Oral Med Oral Pathol Oral Radiol Endod. 1996;81:141-7.

5. Vujevich J, Zirwas M. Treatment of severe, recalcitrant, major aphthous stomatitis with adalimumab. Cutis. 2005;76:129-32.
6. Altenburg A, Zouboulis CC. Current concepts in the treatment of recurrent aphthous stomatitis. Skin Therapy Lett. 2008;13:1-4.

7. Barrons RW. Treatment strategies for recurrent oral aphthous ulcers. Am J Health Syst Pharm. 2001;58:41-50;quiz 1-3.

8. MacPhail L. Topical and systemic therapy for recurrent aphthous stomatitis. Semin Cutan Med Surg. 1997;16:301-7.

9. Scheinfeld N, Rosenberg JD, Weinberg JM. Levamisole in dermatology : a review. Am J Clin Dermatol. 2004;5:97-104.

10. Sun A, Wang JT, Chia JS, Chiang CP. Levamisole can modulate the serum tumor necrosis factor-alpha level in patients with recurrent aphthous ulcerations. J Oral Pathol Med. 2006;35:111-6. 